University of Puget Sound

Sound Ideas

All Faculty Scholarship

Faculty Scholarship

$1-1-1998$

\title{
Brans-dicke Wormholes In The Jordan And Einstein Frames
}

\author{
K. K. Nandi \\ B. Bhattacharjee \\ S. M. K.Alam \\ James C. Evans \\ University of Puget Sound, jcevans@pugetsound.edu
}

Follow this and additional works at: http://soundideas.pugetsound.edu/faculty_pubs

\section{Citation}

Nandi, K. K., B. Bhattacharjee, Smk Alam, and James C. Evans. 1998. "Brans-Dicke wormholes in the Jordan and Einstein frames." Physical Review D 57(2): 823-828.

This Article is brought to you for free and open access by the Faculty Scholarship at Sound Ideas. It has been accepted for inclusion in All Faculty Scholarship by an authorized administrator of Sound Ideas. For more information, please contact soundideas@pugetsound.edu. 


\title{
Brans-Dicke wormholes in the Jordan and Einstein frames
}

\author{
K. K. Nandi, ${ }^{1}$ B. Bhattacharjee, ${ }^{2}$ S. M. K. Alam, ${ }^{1,3}$ and J. Evans ${ }^{4}$ \\ ${ }^{1}$ Department of Mathematics, University of North Bengal, Darjeeling (W.B.), 734 430, India \\ ${ }^{2}$ Department of Physics, University of North Bengal, Darjeeling (W.B.), 734 430, India \\ ${ }^{3} 265 / 3$, West Sheorapara, Mirpur, Dhaka, Bangladesh \\ ${ }^{4}$ Department of Physics, University of Puget Sound, Tacoma, Washington 98416 \\ (Received 22 April 1997; revised manuscript received 6 August 1997; published 30 December 1997)
}

\begin{abstract}
We examine the possibility of static wormhole solutions in the vacuum Brans-Dicke theory both in the original (Jordan) frame and in the conformally rescaled (Einstein) frame. It turns out that, in the former frame, wormholes exist only in a very narrow interval of the coupling parameter, viz., $-3 / 2<\omega<-4 / 3$. It is shown that these wormholes are not traversable in practice. In the latter frame, wormhole solutions do not exist at all unless energy conditions are violated by hand.

[S0556-2821(97)04624-9]
\end{abstract}

PACS number(s): 04.20.Gz, 04.62.+v

\section{INTRODUCTION}

Over the last few years, considerable interest has grown in the field of wormhole physics, following especially the seminal works of Morris, Thorne, and Yurtsever [1,2]. Wormholes are topology changes that connect two asymptotically flat regions. Potential applications of wormhole physics range from the interpretation of gravitational lensing effects to the resolution of several outstanding problems in cosmology [3-5].

In the context of traversable wormholes, a crucial issue is the constraint upon the violation of energy conditions by the stress tensor of quantum or classical fields. There exist several pointwise and average energy conditions [6]. Specifically, for quantum fields, Ford and Roman [7] have proposed, on the basis of certain assumptions, an inequality that constrains the magnitude of the negative energy density at the throat of a traversable wormhole. A fundamental assumption for quantum wormholes is that the stress energy of the spacetime is a renormalized expectation value of the energymomentum operator in some quantum state, say, $|\psi\rangle$. In the literature [8], one actually considers field equations of semiclassical gravity in the form $G_{\mu \nu}=8 \pi\left\langle\psi\left|T_{\mu \nu}\right| \psi\right\rangle$. However, some doubts have been raised, notably by Unruh [9], as to whether field equations in this form could be an exact description of gravity [10]. On the other hand, quantized source fields obey well-defined uncertainty relations and it is expected that uncertainty in the source would induce uncertainty in the gravidynamic variables and in the light cone structure of spacetime $[11,12]$. If the source is taken as $\left\langle T_{\mu \nu}\right\rangle$, such fluctuations would not occur. Despite these questions, it must be emphasized that field equations in the above form provide a very good approximation in many physical situations, especially in the description of the early universe [13].

There also exist classical fields playing the role of "exotic matter" that violates the weak energy condition (WEC), at least at the throat of the wormhole. Examples are provided by the stress-energy tensors occurring in theories where the action contains $\mathbf{R}+\mathbf{R}^{2}$ terms [14], an antisymmetric 3-form axion field coupled to scalar fields [15], and minimally coupled fields with a self-interacting potential [16]. Other theories include string-inspired four-dimensional gravity coupled nonminimally to a scalar field [17], Zee's induced gravity [18], and the Brans-Dicke scalar-tensor theory [19]. Most of the works concentrate on dynamic wormholes, while work on static wormholes is relatively scarce. In particular, in the Brans-Dicke theory, a search for static wormholes has been initiated only recently [20,21], followed by Anchordoqui, Bergliaffa, and Torres [22]. Considering the importance of Brans-Dicke theory in the interpretation of various physical phenomena [23-25] and owing to the fact that, in the limit $\omega \rightarrow \infty$, one recovers general relativity, it is only desirable that a thorough study of classical wormhole solutions be undertaken in this theory.

In this paper, we intend to examine wormhole solutions in the Jordan and Einstein frames which are defined as follows [26]: The pair of variables (metric $g_{\mu \nu}$, scalar $\varphi$ ) defined originally in the Brans-Dicke theory constitute what is called a Jordan frame. Consider now a conformal rescaling

$$
\tilde{g}_{\mu \nu}=f(\varphi) g_{\mu \nu}, \quad \phi=g(\varphi),
$$

such that, in the redefined action, $\phi$ becomes minimally coupled to $\widetilde{g}_{\mu \nu}$ for some functions $f(\varphi)$ and $g(\varphi)$. Then the new pair $\left(\tilde{g}_{\mu \nu}, \phi\right)$ is said to constitute an Einstein frame. There exist different viewpoints as to the question of which of these two frames is physical, but the arguments of Magnano and Sokolowski [26] seem convincing enough in favor of the physicality of the Einstein frame.

In what follows, we shall be concerned only with static spherically symmetric solutions of the Brans-Dicke theory. For this purpose, only a class I type of solution is considered; other classes (II-IV) of solutions can be dealt with in a similar way. Our results are stated as follows. In Sec. II, we consider the Jordan frame and derive the general condition for the existence of wormholes. This condition is then used to find wormhole ranges of $\omega$ in specific cases. Section III shows that these wormholes are not traversable due to the occurrence of a naked singularity. The Einstein frame is con- 
sidered in Sec. IV, and it is shown that wormhole solutions do not exist at all in that frame. The last section, Sec. V, is a summary.

\section{JORDAN FRAME}

In order to investigate the possibility of wormholes in the vacuum (matter-free) Brans-Dicke theory, it is convenient to cast the spacetime metric in the Morris-Thorne canonical form

$$
\begin{gathered}
d \tau^{2}=-e^{2 \Phi(R)} d t^{2}+\left[1-\frac{b(R)}{R}\right]^{-1} d R^{2}+R^{2} d \Omega_{2}^{2}, \\
d \Omega_{2}^{2}=d \theta^{2}+\sin ^{2} \theta d \varphi^{2},
\end{gathered}
$$

where $\Phi(R)$ and $b(R)$ are redshift and shape functions, respectively. These functions are required to satisfy some constraints, enumerated in [1], in order that they represent a wormhole. It is, however, important to stress that the choice of coordinates (Morris-Thorne) is purely a matter of convenience and not a physical necessity. For instance, one could equally well work directly with isotropic coordinates using the analyses of Visser [6], but the final conclusions would be the same. Nonetheless, it must be understood that a more appropriate procedure should involve coordinateindependent proper quantities.

The matter-free action in the Jordan variables is $(G=c$ $=1$ )

$$
S=\frac{1}{16 \pi} \int d^{4} x(-g)^{1 / 2}\left[\varphi \mathbf{R}-\varphi^{-1} \omega(\varphi) g^{\mu \nu} \varphi_{, \mu} \varphi_{, \nu}\right]
$$

The field equations are

$$
\begin{gathered}
\square^{2} \varphi=0, \\
\mathbf{R}_{\mu \nu}-\frac{1}{2} g_{\mu \nu} \mathbf{R}=-\frac{\omega}{\varphi^{2}}\left[\varphi_{, \mu} \varphi_{, \nu}-\frac{1}{2} g_{\mu \nu} \varphi_{, \rho} \varphi^{, \rho}\right] \\
-\frac{1}{\varphi}\left[\varphi_{; \mu ; \nu}-g_{\mu \nu} \square^{2} \varphi\right],
\end{gathered}
$$

where $\square^{2} \equiv\left(\varphi^{; \rho}\right)_{; \rho}$ and $\omega$ is a dimensionless coupling parameter. The general solution, in isotropic coordinates $(r, \theta, \varphi, t)$, is given by

$$
d \tau^{2}=-e^{2 \alpha(r)} d t^{2}+e^{2 \beta(r)} d r^{2}+e^{2 \nu(r)} r^{2} d \Omega_{2}^{2} .
$$

Brans class I solutions [27] correspond to the gauge $\beta-\nu$ $=0$ and are given by

$$
\begin{aligned}
& e^{\alpha(r)}=e^{\alpha_{0}}\left[\frac{1-B / r}{1+B / r}\right]^{1 / \lambda}, \\
& e^{\beta(r)}=e^{\beta_{0}}\left[1+\frac{B}{r}\right]^{2}\left[\frac{1-B / r}{1+B / r}\right]^{(\lambda-C-1) / \lambda}, \\
& \varphi(r)=\varphi_{0}\left[\frac{1-B / r}{1+B / r}\right]^{C / \lambda}
\end{aligned}
$$

$$
\lambda^{2} \equiv(C+1)^{2}-C\left(1-\frac{\omega C}{2}\right)>0
$$

where $\alpha_{0}, \beta_{0}, B, C$, and $\varphi_{0}$ are constants. The constants $\alpha_{0}$ and $\beta_{0}$ are determined by asymptotic flatness condition as $\alpha_{0}=\beta_{0}=0$.

Redefining the radial coordinate $r \rightarrow R$ in the metric (5) as

$$
R=r e^{\beta_{0}}\left[1+\frac{B}{r}\right]^{2}\left[\frac{1-B / r}{1+B / r}\right]^{\Omega}, \quad \Omega=1-\frac{C+1}{\lambda},
$$

we obtain the following functions for $\Phi(R)$ and $b(R)$ :

$$
\begin{gathered}
\Phi(R)=\alpha_{0}+\frac{1}{\lambda}\left[\ln \left\{1-\frac{B}{r(R)}\right\}-\ln \left\{1+\frac{B}{r(R)}\right\}\right], \\
b(R)=R\left[1-\left\{\frac{\lambda\left\{r^{2}(R)+B^{2}\right\}-2 r(R) B(C+1)}{\lambda\left\{r^{2}(R)-B^{2}\right\}}\right\}^{2}\right] .
\end{gathered}
$$

The throat of the wormhole occurs at $R=R_{0}$ such that $b\left(R_{0}\right)=R_{0}$. This gives minimum allowed $r$-coordinate radii $r_{0}^{ \pm}$as

$$
\begin{gathered}
r_{0}^{ \pm}=\alpha^{ \pm} B, \\
\alpha^{ \pm}=(1-\Omega) \pm \sqrt{\Omega(\Omega-2)} .
\end{gathered}
$$

The values $R_{0}^{ \pm}$can be obtained from Eq. (10) using this $r_{0}^{ \pm}$. Noting that $R \rightarrow \infty$ as $r \rightarrow \infty$, we find that $b(R) / R \rightarrow 0$ as $R \rightarrow \infty$. Also, $b(R) / R \leqslant 1$ for all $R \geqslant R_{0}^{ \pm}$. The redshift function $\Phi(R)$ has a singularity at $r=r_{S}=B$. In order that a wormhole be just geometrically traversable, the minimum allowed values $r_{0}^{ \pm}$must exceed $r_{S}=B$. It can be immediately verified from Eq. (10) that $r_{0}^{ \pm} \geqslant B \Rightarrow R_{0}^{ \pm} \geqslant 0$. This is possible only if the range of $\Omega$ is chosen either as $-\infty<\Omega \leqslant 0$ or as $2<\Omega<\infty$. We shall not consider the latter range here. $[1]$

The energy density of the wormhole material is given by

$$
\rho(R)=\left(8 \pi R^{-2}\right)(d b / d R),
$$

and a straightforward calculation gives

$$
\begin{aligned}
d b / d R & =4 r^{2}(R) B^{2}\left[r^{2}(R)-B^{2}\right]^{-2} \Omega(2-\Omega) \\
& =4 r^{2}(R) B^{2}\left[r^{2}(R)-B^{2}\right]^{-2}\left[1-\left(\frac{C+1}{\lambda}\right)^{2}\right] .
\end{aligned}
$$

Therefore, the most general condition for the violation of the WEC is that

$$
C(\omega)+1>\lambda(\omega),
$$

where the real function $C(\omega)$ is as yet unspecified. As long as the general condition (17), which ensures $R_{0}^{ \pm}>0$, is satisfied, it follows that

$$
b_{0}^{\prime}=\left.\frac{d b}{d R}\right|_{R=R_{0}^{ \pm}}=-1
$$


so that $\rho_{0}=\left.\rho\right|_{R=R_{0}^{ \pm}}<0$, and a violation of the WEC at the throat is achieved thereby. In the limit $r_{0}^{ \pm} \rightarrow B+$, or, equivalently, $R_{0}^{ \pm} \rightarrow 0+$, one obtains $\rho_{0} \rightarrow-\infty$. This means that there occurs an infinitely large concentration of exotic matter at the throat when its $r$ radius is in the vicinity of the Schwarzschild radius $r_{s}=B$. No upper limit to this classical negative energy density is known to us. The general profile for $\rho(R)$ for a given wormhole configuration is that $\rho(R)$ attains its maximum at the throat and falls off in an inverse square law as one moves away from the throat to the asymptotic region.

The constraint (17) can be rephrased, using Eq. (9), as

$$
C(\omega)\left[1-\frac{\omega C(\omega)}{2}\right]>0,
$$

and depending on the form of $C(\omega)$, this inequality fixes the range of wormhole values of $\omega$, provided one excludes the forbidden range coming from the requirement that $\lambda^{2}>0$. A further exclusion of the range $\omega \leqslant-3 / 2$ comes from a "physical" requirement that the theory be transferrable to Einstein frame [26]. In the limiting case, $C(\omega) \rightarrow 0$, $\lambda(\omega) \rightarrow 1$ as $\omega \rightarrow \infty$, one simply recovers the Schwarzschild exterior metric in standard coordinates from Eqs. (11) and (12), so that $b(R)=2 M$ and $b_{0}^{\prime}=0$. The inequality (19) is violated, and there occurs no traversable wormhole, as is well known [1].

The analysis of Agnese and La Camera [20] corresponds, as pointed out earlier [21], to the choice

$$
C(\omega)=-\frac{1}{\omega+2},
$$

which suggests, via Eq. (19), a wormhole range $\omega<-4 / 3$. The forbidden range turns out to be $-2<\omega<-3 / 2$, which is already a part of the unphysical range $\omega \leqslant-3 / 2$. Therefore, one is left with a very narrow actual interval for wormhole solutions, viz., $-3 / 2<\omega<-4 / 3$. It appears that the authors just missed this interval.

We should recall here that Eq. (20) is derived on the basis of a weak field (post Newtonian) approximation and there is no reason for Eq. (20) to hold for stars with a strong field such as neutron stars. In reality, if we assume such a restriction as Eq. (20), the junction conditions for the metric and scalar field are not satisfied at the boundary of the stars [28]. Evidently, any form for $C(\omega)$ different from Eq. (20) would lead to a different wormhole interval for $\omega$. For example, in the context of gravitational collapse in the Brans-Dicke theory, Matsuda [28] chose $C(\omega) \propto-\omega^{-1 / 2}$. Let us take $C(\omega)=-q \omega^{-1 / 2}$ and choose $q<0$ such that $C(\omega)>0$. Then the constraint (19) will be satisfied only if $\omega>4 / q^{2}$. The exact form of $C(\omega)$ should be known a a priori from other physical considerations. However, this is just a tentative example and is meant to highlight how crucially the wormhole range for $\omega$ depends on the form of $C(\omega)$.

The constraint (17) is based only on the requirement of geometric traversability, i.e., on the requirement that the throat radii be larger than the event horizon radius $r=B$. Therefore, an immediate inquiry is whether such wormholes are traversable in practice. We discuss this issue in the following section.

\section{TRAVERSABILITY}

In order to get a firsthand idea about traversability in the Jordan frame, a convenient procedure is to calculate the scales over which wormhole functions change. Ford and Raman [7] defined the following quantities at the throat $R$ $=R_{0}$ of a traversable wormhole:

$$
\overline{r_{0}}=R_{0}, \quad r_{1}=\frac{R_{0}}{\left|b_{0}^{\prime}\right|}, \quad R_{2}=\frac{1}{\left|\Phi_{0}^{\prime}\right|}, \quad r_{3}=\left|\frac{\Phi_{0}^{\prime}}{\Phi_{0}^{\prime \prime}}\right| .
$$

These quantities are a measure of coordinate length scales at the throat over which the functions $b(R), \Phi(R)$, and $\Phi^{\prime}(R)$ change, respectively. For the class I solutions, they become

$$
\overline{r_{0}}=R_{0}^{ \pm}, \quad r_{1}=R_{0}^{ \pm}, \quad R_{2}=0, \quad r_{3}=0 .
$$

The vanishing of $R_{2}$ and $r_{3}$ implies that both $\Phi(R)$ and $\Phi^{\prime}(R)$ exhibit an abrupt jump at the throat. It is therefore expected that the tidal forces at the throat would be large. That this is indeed so can be verified by calculating, for example, the differential of the radial tidal acceleration [1] given in an orthonormal frame $\left(\hat{e}_{t}, \hat{e}_{R}, \hat{e}_{\theta}, \hat{e}_{\varphi}\right)$ by

$$
\Delta a^{R}=-\mathbf{R}_{\hat{R} \hat{t} \hat{R} \hat{t}} \xi^{R},
$$

where $\xi^{R}$ is the radial component of the separation vector and

$$
\left|\mathbf{R}_{\hat{R} \hat{t} \hat{R} \hat{t}}\right|=\left|(1-b / R)\left[-\Phi^{\prime \prime}+\frac{b^{\prime} R-b}{2 R(R-b)} \Phi^{\prime}-\left(\Phi^{\prime}\right)^{2}\right]\right| .
$$

For the metric given by Eqs. (11) and (12), we find

$$
\begin{aligned}
\left|\mathbf{R}_{\hat{R} \hat{t} \hat{R} \hat{t}}\right|= & \mid \frac{B r}{\lambda R^{2}\left(r^{2}-B^{2}\right)}\left[2(1-b / R)^{1 / 2}+(1-b / R)^{-1 / 2} b^{\prime}\right. \\
& \left.+\frac{2 \lambda\left(r^{2}+B^{2}\right)-4 B r}{\lambda\left(r^{2}-B^{2}\right)}\right] \mid
\end{aligned}
$$

At the throat where $b\left(R_{0}^{ \pm}\right)=R_{0}^{ \pm}$, we have $\left|\mathbf{R}_{\hat{R} \hat{t} \hat{R} \hat{t}}\right| \rightarrow \infty$, and this implies $\Delta a^{R} \rightarrow \infty$. As we march away from the throat to the asymptotic limit $r \rightarrow \infty$ or, $R \rightarrow \infty$, we find $\left|\mathbf{R}_{\hat{R} \hat{t} \hat{R} t}\right| \rightarrow 0$, as is to be expected.

Such an infinitely large tidal force at the throat is presumably related to the presence of singular null surface or naked singularity in the wormhole spacetime. These wormholes, to use a phrase by Visser [6], are "badly diseased."

The occurrence of singular null surface in the scalartensor theories is directly related to the "no-hair theorem," which commonly means that "black holes have no scalar hair" [29]. Early investigations into the no-hair theorem in the Brans-Dicke theory are due to Hawking [30], Chase [31], Teitelboim [32], and Bekenstein [33]. Recently, Saa [34] has formulated a new no-hair theorem which basically relies on the assessment of the behavior of scalar curvature $\mathbf{R}$, which, for the metric (6) and (7), turns out to be

$$
\mathbf{R}(r)=\frac{4 \omega C^{2} B^{2} r^{4}(r+B)^{2 \Omega-6}}{\lambda^{2}(r-B)^{2 \Omega+2}} .
$$


Then it follows that $\mathbf{R} \rightarrow \infty$ as $r \rightarrow B+$ for $C \neq 0$. In other words, the scalar curvature diverges as $R \rightarrow 0+$, implying that this shrunk surface does not represent a black hole for $\varphi \neq$ const. It is instead a naked singularity [34]. On the other hand, if $C \rightarrow 0$ and $\lambda \rightarrow 1$, we have a finite value of $\mathbf{R}$ as $r \rightarrow B$. This means that we have a black hole solution for $\varphi$ $=$ const, in total accordance with the no-hair theorem.

Generally speaking, wormhole solutions obtain in the Jordan frame because the sign of the energy density is indefinite in that frame. The sign is positive or negative according as $C(\omega)+1<\lambda$ or $C(\omega)+1>\lambda$. Let us examine the situation in the Einstein frame, defined earlier.

\section{EINSTEIN FRAME}

Under the conformal transformation

$$
\widetilde{g}_{\mu \nu}=p g_{\mu \nu}, \quad p=\frac{1}{16 \pi} \varphi
$$

and a redefinition of the Brans-Dicke scalar

$$
d \phi=\left(\frac{\omega+\frac{3}{2}}{\alpha}\right)^{1 / 2} \frac{d \varphi}{\varphi}
$$

in which we have intentionally introduced an arbitrary parameter $\alpha$, the action (3) in the Einstein variables $\left(\widetilde{g}_{\mu \nu}, \phi\right)$ becomes

$$
S=\int d^{4} x(-\widetilde{g})^{1 / 2}\left[\widetilde{\mathbf{R}}-\alpha \widetilde{g}^{\mu \nu} \phi_{, \mu} \phi_{, \nu}\right] .
$$

The field equations are

$$
\begin{gathered}
\widetilde{\mathbf{R}}_{\mu \nu}=\alpha \phi_{, \mu} \phi_{, \nu}, \\
\square^{2} \phi=0 .
\end{gathered}
$$

The solutions of Eqs. (30) and (31) can be obtained, using the transformations (27) and (28), as

$$
\begin{gathered}
d \tau^{2}=-\left(1+\frac{B}{r}\right)^{2 \beta}\left(1-\frac{B}{r}\right)^{-2 \beta} d t^{2}+\left(1-\frac{B}{r}\right)^{2(1-\beta)} \\
\times\left(1+\frac{B}{r}\right)^{2(1+\beta)}\left[d r^{2}+r^{2} d \Omega_{2}^{2}\right] \\
\phi=\left[\left(\frac{\omega+\frac{3}{2}}{\alpha}\right)\left(\frac{C^{2}}{\lambda^{2}}\right)\right]^{1 / 2} \ln \left[\frac{1-B / r}{1+B / r}\right] \\
\beta=\frac{1}{\lambda}\left(1+\frac{C}{2}\right) .
\end{gathered}
$$

The expression for $\lambda^{2}$, of course, continues to be the same as Eq. (9), and using this, we can rewrite Eq. (33) as

$$
\phi=\left[\frac{2\left(1-\beta^{2}\right)}{\alpha}\right]^{1 / 2} \ln \left[\frac{1-B / r}{1+B / r}\right] .
$$

Casting the metric (32) into the Morris-Thorne form, we can find the wormhole throat $r$ radii to be

$$
r_{0}^{ \pm}=B\left[\beta \pm\left(\beta^{2}-1\right)^{1 / 2}\right] .
$$

For real $r_{0}^{ \pm}$, we must have $\beta^{2} \geqslant 1$. But $\beta^{2}=1$ corresponds to a nontraversable wormhole since $r_{0}^{ \pm}$coincides with the singular radius $r_{S}=B$. From Eq. (35), it follows that, if $\alpha>0$ and $\beta^{2}>1$, then no wormhole is possible as $\phi$ becomes imaginary. This result is quite consistent with the fact that the stress-energy tensor for massless minimally coupled scalar field $\phi$ : viz.,

$$
T_{\mu \nu}=\alpha\left(\phi_{, \mu} \phi_{, \nu}-\frac{1}{2} \widetilde{g}_{\mu \nu} \phi^{, \sigma} \phi_{, \sigma}\right)
$$

satisfies all energy conditions [6]. The Einstein frame is thus called "physical" for which the restriction $\omega>-3 / 2$ follows from Eq. (33).

On the other hand, if we choose $\alpha<0$, which amounts to violating all energy conditions by brute force, one may find wormholes for $\beta^{2}>1$ in Eq. (35) or, equivalently, for $\omega<$ $-3 / 2$.

We wish to point out a few more relevant points.

(i) Just as in the Jordan frame, the "no-hair theorem", holds also in the Einstein frame. This can be seen from the expression for scalar curvature $\widetilde{\mathbf{R}}$ computed from the metric (32):

$$
\widetilde{\mathbf{R}}=\frac{8 B^{2} r^{4}\left(1-\beta^{2}\right)}{(r-B)^{2(2-\beta)}(r+B)^{2(2+\beta)}}
$$

One can see that $\widetilde{\mathbf{R}}$ is negative for wormhole solutions. In the Schwarzschild limit $\beta \rightarrow 1, \widetilde{\mathbf{R}}$ is finite for $r \rightarrow B$, and a black hole solution results, in complete accordance with the nohair theorem [34]. The divergence of $\phi$ at $r=B$ has been shown to be physically innocuous $[35,36]$. Generally, for $\beta$ $\neq 1, \widetilde{\mathbf{R}} \rightarrow \infty$ as $r \rightarrow B$. This implies that the surface $r=B$ (or, $R=0$ ) is not a black hole surface for nonconstant $\phi$. This conclusion is in agreement with that reached by Agnese and La Camera [37] in a different way.

(ii) The Arnowitt-Deser-Misner (ADM) mass of the configuration is defined by

$$
M=\frac{1}{16 \pi} \lim _{S \rightarrow \infty} \int_{S} \sum_{i, j=1}^{3}\left(\partial_{j} g_{i j}-\partial_{i} g_{j j}\right) n^{i} d S,
$$

where $S$ is a 2 -surface enclosing the active region and $n^{i}$ denotes the unit outward normal. For the metric (32), we get

$$
M=2 B \beta,
$$

and using this value, the metric can be expanded in the weak field as

$$
\begin{aligned}
d \tau^{2}= & -\left(1+2 M r^{-1}+\cdots\right) d t^{2}+\left(1-2 M r^{-1}+2 M r^{-2}\right. \\
& +\cdots)\left[d r^{2}+r^{2} d \Omega_{2}^{2}\right]
\end{aligned}
$$

that is, it predicts exactly the same results for a neutral test particle as does Einstein's general relativity. The factor $\alpha$ does not appear in the metric, although it does appear in the scalar field $\phi$. Hence, $\alpha$ cannot be determined by any metric test of gravity.

(iii) It should be remarked that if we replace $B$ by another integration constant $m / 2$, the solutions (32) and (35) become 
those proposed by Buchdahl [38] long ago. Defining the field strength $\sigma$ for the scalar field $\phi$ in analogy with an "electrostatic field," one obtains

$$
\sigma=-2 \delta m, \quad \delta=\left[\left(1-\beta^{2}\right) / 2 \alpha\right]^{1 / 2} .
$$

Then, from Eqs. (40) and (42), it follows that the gravitation producing mass $M$ is given by

$$
M^{2}=m^{2}-\frac{1}{2} \alpha \sigma^{2},
$$

where $m$ can be regarded as the strength of the source excluding the scalar field. For $\beta \rightarrow 0$, we have $M \rightarrow 0$. The situation in this case is that, for $\alpha>0$, we can have both $m$ and $\sigma$ nonzero, but with their effects mutually anulled. In other words, we obtain a configuration which is indifferent to a gravitational interaction with distant bodies. The reason is that the stresses of the $\phi$ field contribute an amount of negative gravitational potential energy (attractive) just sufficient to make the total energy zero [38]. On the other hand, if $\alpha$ $<0$, the $\phi$ field has a positive gravitational potential energy (repulsive). We cannot take $\beta \rightarrow 0$ owing to Eq. (42), but it is possible to make $m \rightarrow 0$ so that $M \rightarrow 0$. In this case, we have $\sigma=0$. That is, the vanishing of total energy implies a vanishing of individual source contributions.

\section{SUMMARY}

The foregoing analysis reveals that spherically symmetric static vacuum Brans-Dicke wormholes exist in the Jordan frame only in a very narrow interval $-3 / 2<\omega<-4 / 3$, corresponding to a physical situation where the post-Newtonian approximation is valid. In general, the wormhole range for $\omega$ depends entirely on the form of $C(\omega)$ supposed to be dictated by physical conditions. Wormhole solutions do not exist at all in the conformally rescaled (Einstein) frame unless one is willing to violate the energy conditions by choice ( $\alpha$ $<0$ ). However, such a manipulation is not always necessary.
For example, there exist theories where one adds to the Einstein frame vacuum action other fields (such as the axion field [15]) or potentials [39] and obtains dynamic wormhole solutions in a natural way.

It is evident that the factor $\alpha$ does not appear in the metric (32), although it does appear in the expression for the scalar field $\phi$. In particular, for local tests of gravity, the predictions are exactly the same as those of Einstein's general relativity where the Robertson parameters take on values $\alpha=\beta=\gamma$ $=1$. In contrast, in the Jordan frame, one has $\alpha=\beta=1, \gamma$ $=(\omega+1) /(\omega+2)$. For finite $\omega$, it is evident that the predictions deviate somewhat from the actually observed values.

The Arnowitt-Deser-Misner (ADM) mass of the configuration is positive in both the frames. In the Jordan frame, it is $M=(2 B / \lambda)(C+1)$, while in the Einstein frame it is $M$ $=2 B \beta$. It is also shown that a gravitationally indifferent real configuration with zero total energy $(M=0)$ does or does not exist in the Einstein frame according as $\alpha>0$ or $\alpha<0$.

An interesting feature of Brans-Dicke wormholes is that infinitely large radial tidal accelerations occur at the throat so that these wormholes are not traversable in practice. This feature is reflected in the absence of a black hole surface at $r=B$ or, in the Morris-Thorne coordinates, at $R=0$.

We have not addressed the question of stability of BransDicke wormholes in this paper. With regard to classical perturbations, it should be pointed out that the results of Anchordoqui, Bergliaffa, and Torres [22] indicate that addition of extra ordinary matter does not destroy the wormhole. The effect of the quantum back reaction of the scalar field on stability will be considered elsewhere.

\section{ACKNOWLEDGMENTS}

One of us (S.M.K.A.) would like to thank the Indian Council for Cultural Relations (ICCR), New Delhi, for financial support under an Exchange Program of the Government of India.
[1] M. S. Morris and K. S. Thorne, Am. J. Phys. 56, 395 (1988).

[2] M. S. Morris, K. S. Thorne, and U. Yurtsever, Phys. Rev. Lett. 61, 1446 (1988).

[3] J. G. Cramer et al., Phys. Rev. D 51, 3117 (1995).

[4] D. Hochberg and T. W. Kephart, Gen. Relativ. Gravit. 26, 219 (1994); Phys. Lett. B 268, 377 (1991); Phys. Rev. Lett. 70, 2665 (1993).

[5] S. Coleman, Nucl. Phys. B310, 643 (1988); B307, 867 (1988); S. W. Hawking, ibid. B335, 155 (1990); S. Giddings and A. Strominger, ibid. B321, 481 (1988).

[6] M. Visser, Phys. Rev. D 39, 3182 (1989); Nucl. Phys. B328, 203 (1989); Lorentzian Wormholes-From Einstein To Hawking (AIP, New York, 1995).

[7] L. H. Ford and T. A. Roman, Phys. Rev. D 51, 4277 (1995); 53, 5496 (1996); 53, 1988 (1996); 55, 2082 (1997).

[8] D. Hochberg, A. Popov, and S. V. Sushkov, Phys. Rev. Lett. 78, 2050 (1997); F. Schein and P. C. Aichelberg, ibid. 77, 4130 (1996).

[9] W. G. Unruh, in Quantum Theory of Gravity, edited by S. M.
Christensen (Hilger, Bristol, 1984).

[10] D. N. Page and C. D. Geilker, Phys. Rev. Lett. 47, 979 (1981).

[11] T. Padmanabhan, T. R. Seshadri, and T. P. Singh, Int. J. Mod. Phys. A 1, 491 (1986).

[12] K. K. Nandi, A. Islam, and J. Evans, Int. J. Mod. Phys. A 12, 3171 (1997).

[13] L. Parker and S. A. Fulling, Phys. Rev. D 7, 2357 (1973).

[14] D. Hochberg, Phys. Lett. B 251, 349 (1990).

[15] D. H. Coule and K. Maeda, Class. Quantum Grav. 7, 955 (1990); P. F. Gonzalez-Diaz, Phys. Lett. B 233, 85 (1989); H. Fukutaka, K. Ghoruku, and K. Tanaka, ibid. 222, 191 (1989).

[16] S. Cotsakis, P. Leach, and G. Flessas, Phys. Rev. D 49, 6489 (1994).

[17] S. Giddings and A. Strominger, Nucl. Phys. B306, 890 (1988).

[18] A. Zee, Phys. Rev. Lett. 42, 417 (1979).

[19] F. S. Accetta, A. Chodos, and B. Shao, Nucl. Phys. B333, 221 (1990).

[20] A. G. Agnese and M. La Camera, Phys. Rev. D 51, 2011 (1995). 
[21] K. K. Nandi, A. Islam, and J. Evans, Phys. Rev. D 55, 2497 (1997)

[22] L. Anchordoqui, S. P. Bergliaffa, and D. F. Torres, Phys. Rev. D 55, 5226 (1997).

[23] N. Riazi and H. R. Askari, Mon. Not. R. Astron. Soc. 261, 229 (1993).

[24] K. K. Nandi and A. Islam, Ind. J. Phys. B 68, 539 (1994).

[25] T. Harada et al., Phys. Rev. D 55, 2024 (1997).

[26] G. Magnano and L. M. Sokolowski, Phys. Rev. D 50, 5039 (1994).

[27] C. H. Brans, Phys. Rev. 125, 2194 (1962).

[28] T. Matsuda, Prog. Theor. Phys. 47, 738 (1972).

[29] R. Ruffini and J. A. Wheeler, Phys. Today 24(1), 30 (1971).
[30] S. W. Hawking, Commun. Math. Phys. 25, 167 (1972).

[31] J. E. Chase, Commun. Math. Phys. 19, 276 (1970).

[32] C. Teitelboim, Lett. Nuovo Cimento 3, 326 (1972).

[33] J. D. Bekenstein, Phys. Rev. Lett. 28, 452 (1972).

[34] A. Saa, J. Math. Phys. 37, 2346 (1996).

[35] T. Zannias, J. Math. Phys. 36, 6970 (1995).

[36] J. D. Bekenstein, Ann. Phys. (N.Y.) 91, 72 (1975); 82, 535 (1974).

[37] A. G. Agnese and M. La Camera, Phys. Rev. D 31, 1280 (1985).

[38] H. A. Buchdahl, Phys. Rev. 115, 1325 (1959).

[39] J. D. Bekenstein, Phys. Rev. D 51, R6608 (1995). 